\title{
The Two-scale Asymptotic Expansion for One Class of Thermoelastic Problem in Periodic Composite with Boundary Layer Xiao-Jun DENG ${ }^{1,2, a}$, Ming-Xiang DENG ${ }^{3, b, *}$ and Yong-Ping FENG ${ }^{3, c}$
}

\author{
${ }^{1}$ Business School of Sichuan University, 610064 Chengdu, China \\ ${ }^{2}$ School of Management, Northwest University of Nationalities, 730070 Lanzhou, China \\ ${ }^{3}$ School of Mathematics and Information Sciences, Guangzhou University, \\ 510006 Guangzhou, China

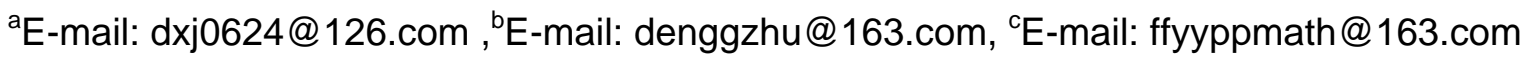 \\ ${ }^{*}$ Corresponding author
}

Keywords: Thermoelastic problem, Two-scale method, Periodic composite, Boundary layer

\begin{abstract}
The thermoelastic materials and functional composites are used in some intelligent equipment in some special fields. The two-scale asymptotic expansion for partial differential equations of thermoelstic problem in periodic structure with boundary layer is constructed, and the two-scale asymptotic error estimations corresponding to the asymptotic solution is analyzed.
\end{abstract}

\section{Introduction}

In the developments of thermodynamics and intelligent materials, we usually need to develop some thermal composites and functional materials. In recent years, many scholars have concentrated on these materials. For many engineers, their main interests are the effective physical behavior of the thermal-mechanical and physical phenomenon. In mathematics, many researchers have discussed the numerical simulation methods and finite element method to simulate these composites [1-4]. Owing to the small periodicity and coupling effects in multiple physical fields, it is very difficult to obtain the analytic solutions for these problems. Thus the effective mechanical and physical properties of these materials are the main research directions for scholars. Up to now, the results of approximation solutions for general coupling thermoelastic problem in multiple fields with boundary layer are very few. In this paper, by means of multi-scale method, the two-scale asymptotic expansion and the two-scale asymptotic error estimation for one class of coupling thermoelastic problem in periodic structure with boundary layer are analyzed.

The general linear thermoelastic problem in periodic domain with boundary layer in mathematics could be described by the following boundary value problem,

$$
\left\{\begin{array}{l}
\frac{\partial}{\partial x_{i}}\left(k_{i j}^{\varepsilon} \frac{\partial \theta^{\varepsilon}}{\partial x_{j}}\right)-c^{\varepsilon} \theta^{\varepsilon}+h^{\varepsilon}=0, \Omega^{\varepsilon}, \\
\frac{\partial}{\partial x_{j}}\left(a_{i j h k}^{\varepsilon} \varepsilon_{h k}\left(\mathbf{u}^{\varepsilon}\right)\right)-\frac{\partial}{\partial x_{j}}\left(a_{i j h k}^{\varepsilon} b_{h k}^{\varepsilon} \theta^{\varepsilon}\right)-d_{i}^{\varepsilon} u_{i}^{\varepsilon}+f_{i}^{\varepsilon}(x)=0, \Omega^{\varepsilon}, \\
\theta^{\varepsilon}(x)=\theta_{0}(x), \mathbf{u}^{\varepsilon}(x)=\mathbf{u}_{0}(x), \text { on } \Gamma_{\varepsilon} .
\end{array}\right.
$$

Here $\omega$ is an unbounded periodic domain in $R^{n}, \Omega$ is a bounded domain in $R^{n}$, and $\Omega^{\varepsilon}=\Omega \bigcap \omega=\Omega_{1}^{\varepsilon} \cup \Omega_{2}^{\varepsilon}$ is a periodic domain with boundary layer, $\Omega_{1}^{\varepsilon}$ is the periodic part and $\Omega_{2}^{\varepsilon}$ is the boundary layer part, and $\Omega_{2}^{\varepsilon}=\left\{x \mid \varepsilon \leq \sup _{y \in \Omega^{\varepsilon}}(\operatorname{dist}|x-y|) \leq 2 \varepsilon\right\}, \partial \Omega^{\varepsilon}=\Gamma_{\varepsilon}$ satisfies the Lipschitz boundary condition; $\mathbf{u}^{\varepsilon}(x)$ and $\theta^{\varepsilon}(x)$ denote the 
displacement vector and the increment of the temperature as compared with the reference temperature, respectively; $\left\{a_{i j h k}^{\varepsilon}\right\},\left\{b_{h k}^{\varepsilon}\right\}$ and $\left\{k_{i j}^{\varepsilon}\right\}$ are the stiffness, thermal expansion and thermal conductivity of materials, respectively, and these tensors satisfy $\varepsilon\left(k_{1}, k_{2}\right)$ and $p\left(\lambda_{1}, \lambda_{2}\right)$ conditions in [1], $c^{\varepsilon}$ and $d_{i}^{\varepsilon}$ denote the 1st-order modification of thermal conductivity and the stiffness; $\mathbf{f}^{\varepsilon}(x)$ and $h^{\varepsilon}(x)$ represent the internal force and internal heat sources, respectively. For simplicity, it is assumed that $\mathbf{f}^{\varepsilon}, h^{\varepsilon}, \theta_{0}$ and $\mathbf{u}_{0}$ are sufficiently smooth vector or scalar functions in $\Omega^{\varepsilon} \cdot \varepsilon_{h k}\left(\mathbf{u}^{\varepsilon}\right)$ are the strains evaluated from the displacement $\mathbf{u}^{\varepsilon}(x)$, and

$$
\varepsilon_{h k}\left(\mathbf{u}^{\varepsilon}\right)=\frac{1}{2}\left(\frac{\partial u_{h}^{\varepsilon}}{\partial x_{k}}+\frac{\partial u_{k}^{\varepsilon}}{\partial x_{h}}\right)
$$

\section{The Two-scale Formal Asymptotic Expression}

Owing to the small periodicity and coupling effects in multiple physical fields, it is very difficult to obtain the analytic solution for Eq.1. There are some traditional methods to simulate the homogenization constants for Eq.1, such as averaging method and homogenization method. But these methods failed to capture the local strains and stresses behavior in detail. So as to capture the local coupling effects let us turn to discuss the formal asymptotic solution corresponding to Eq.1.

Assuming that in $\boldsymbol{\Omega}_{1}^{\varepsilon},\left(\mathbf{u}^{\varepsilon}(x), \theta^{\varepsilon}(x)\right)$ could be expanded formally as follows,

$$
\left\{\begin{array}{c}
\theta^{\varepsilon}(x)=\theta^{0}(x)+\varepsilon H_{\alpha_{1}}(\xi) \frac{\partial \theta^{0}(x)}{\partial x_{\alpha_{1}}}+\varepsilon^{2} H_{\alpha_{1} \alpha_{2}}(\xi) \frac{\partial^{2} \theta^{0}(x)}{\partial x_{\alpha_{1}} \partial x_{\alpha_{2}}}+\varepsilon^{3} H_{\alpha_{1} \alpha_{2} \alpha_{3}}(\xi) \frac{\partial^{3} \theta^{0}(x)}{\partial x_{\alpha_{1}} \partial x_{\alpha_{2}} \partial x_{\alpha_{3}}}+\ldots, \\
\mathbf{u}^{\varepsilon}(x)=\mathbf{u}^{0}(x)+\varepsilon \mathbf{N}_{\alpha_{1}}(\xi) \frac{\partial \mathbf{u}^{0}(x)}{\partial x_{\alpha_{1}}}+\varepsilon^{2} \mathbf{N}_{\alpha_{1} \alpha_{2}}(\xi) \frac{\partial^{2} \mathbf{u}^{0}(x)}{\partial x_{\alpha_{1}} \partial x_{\alpha_{2}}}+\varepsilon \mathbf{M}_{0}(\xi) \theta^{0}(x)+\varepsilon^{2} \mathbf{M}_{\alpha_{1}}(\xi) \frac{\partial \theta^{0}(x)}{\partial x_{\alpha_{1}}}+\ldots
\end{array}\right.
$$

where $H_{\alpha}(\xi), \mathbf{N}_{\alpha}(\xi), \mathbf{M}_{\alpha}(\xi)$ are the 1-periodic scalar, matrix and vector functions defined on $R^{n}$, respectively, which are solutions of some special partial differential equations defined on unit cube $Q$.

Substituting Eq. 2 into Eq. 1, by means of detail and careful deducting and comparing the coefficients of power $\varepsilon^{-1}, \varepsilon^{0}, \varepsilon^{1}, \ldots$ on both sides of Eq. 1, we could define these unknowns cell functions $H_{\alpha}(\xi), \mathbf{N}_{\alpha}(\xi), \mathbf{M}_{\alpha}(\xi)$ one by one, recursively. From Eq. 1, Eq.2 and the coefficients of $\varepsilon^{-1}, \mathbf{N}_{\alpha_{1} m}(\xi), \mathbf{M}_{0}(\xi)$ could be determined in $Q$ by the following equations:

$$
\begin{gathered}
\begin{cases}\frac{\partial}{\partial \xi_{j}}\left[a_{i j h k}(\xi) \varepsilon_{h k}\left(\mathbf{N}_{\alpha_{1} m}(\xi)\right)\right]=-\frac{\partial}{\partial \xi_{j}}\left(a_{i j m \alpha_{1}}(\xi)\right) & \xi \in Q \\
\mathbf{N}_{\alpha_{1} m}(\xi)=0 & \xi \in \partial Q\end{cases} \\
\begin{cases}\frac{\partial}{\partial \xi_{j}}\left[a_{i j h k}(\xi) \varepsilon_{h k}\left(\mathbf{M}_{0}(\xi)\right)\right]=\frac{\partial}{\partial \xi_{j}}\left(a_{i j h k}(\xi) b_{h k}(\xi)\right) & \xi \in Q \\
\mathbf{M}_{0}(\xi)=0 & \xi \in \partial Q\end{cases}
\end{gathered}
$$

Similarly, $\mathbf{N}_{\alpha_{1} \alpha_{2} m}(\xi), \mathbf{M}_{\alpha_{1}}(\xi)$ could be determined by the following equations: 


$$
\left\{\begin{array}{l}
\frac{\partial}{\partial \xi_{j}}\left(a_{i j h k}(\xi) \varepsilon_{h k}\left(\mathbf{N}_{\alpha_{1} \alpha_{2}}(\xi)\right)\right)=-a_{i \alpha_{2} m \alpha_{1}}(\xi)-a_{i \alpha_{2} h k}(\xi) \frac{\partial}{\partial \xi_{k}} \mathbf{N}_{\alpha_{1} h m}(\xi)-\frac{\partial}{\partial \xi_{j}}\left(a_{i j h \alpha_{2}}(\xi) \mathbf{N}_{\alpha_{1} h m}(\xi)\right) \\
\quad+\hat{a}_{i j k h}, i=1,2, . . n, \xi \in Q \\
\mathbf{N}_{\alpha_{1} \alpha_{2}}(\xi)=0, \quad \xi \in \partial Q
\end{array}\right.
$$

and

$$
\left\{\begin{array}{c}
\frac{\partial}{\partial \xi_{j}}\left(a_{i j h k}(\xi) \varepsilon_{h k}\left(\mathbf{M}_{\alpha_{1}}(\xi)\right)\right)=-a_{i \alpha_{1} h k}(\xi) \varepsilon_{h k}\left(\mathbf{M}_{0}(\xi)\right)-\frac{\partial}{\partial \xi_{j}}\left(a_{i j h \alpha_{1}}(\xi) \mathbf{M}_{0 h}(\xi)\right)+a_{i \alpha_{1} h k}(\xi) b_{h k}(\xi) \\
+\frac{\partial}{\partial \xi_{j}}\left(a_{i j h k}(\xi) b_{h k}(\xi) H_{\alpha_{1}}(\xi)\right)+\hat{b}_{i j}, \quad \xi \in Q \\
\mathbf{M}_{\alpha_{1}}(\xi)=0, \quad \xi \in \partial Q
\end{array}\right.
$$

where

$$
\begin{gathered}
\hat{a}_{i j h k}=\int_{Q}\left[a_{i j h k}(\xi)+a_{i j l m}(\xi) \varepsilon_{l m}\left(\mathbf{N}_{h k}(\xi)\right)\right] d \xi, \\
\hat{b}_{i j}=\int_{Q}\left[a_{i j h k}(\xi) \varepsilon_{h k}\left(\mathbf{M}_{0}(\xi)\right)-a_{i j h k}(\xi) b_{h k}(\xi)\right] d \xi,
\end{gathered}
$$

are the homogenization constants. $H_{\alpha}(\xi)$ could be defined in unit cell $Q$ in a similar way. $\left(\mathbf{u}^{0}(x), \theta^{0}(x)\right)$ is the homogenization solution corresponding to $\left(\mathbf{u}^{\varepsilon}(x), \theta^{\varepsilon}(x)\right)$, and it can be determined by the following equations with homogenization constants that reflect the effective properties of composites,

$$
\left\{\begin{array}{l}
\frac{\partial}{\partial x_{i}}\left(\hat{k}_{i j} \frac{\partial \theta^{0}}{\partial x_{j}}\right)-\hat{c} \theta^{0}=-\hat{h}(x), x \in \Omega, \\
\frac{\partial}{\partial x_{j}}\left(\hat{a}_{i j h k} \varepsilon_{h k}\left(\mathbf{u}^{0}\right)\right)-\hat{d}_{i} u_{i}^{0}=-\hat{f}_{i}(x)+\frac{\partial}{\partial x_{j}}\left(\hat{b}_{i j} \theta^{0}\right), i=1,2, \ldots, n, x \in \Omega, \\
\mathbf{u}^{0}(x)=\mathbf{u}_{0}(x), \theta^{0}(x)=\theta_{0}(x), x \in \partial \Omega .
\end{array}\right.
$$

Where

$$
\begin{aligned}
& \hat{k}_{i j}=\int_{Q}\left[k_{i j}(\xi)+k_{i l}(\xi) \frac{\partial H_{j}(\xi)}{\partial \xi_{l}}\right] d \xi, \\
& \hat{c}=\int_{Q} c(\xi) d \xi, \hat{d}_{i}=\int_{Q} d_{i}(\xi) d \xi, \hat{f}_{i}(x)=\int_{Q} f_{i}(x, \xi) d \xi, \hat{h}(x)=\int_{Q} h(x, \xi) d \xi,
\end{aligned}
$$

In practice, $\left(\mathbf{u}^{\varepsilon}(x), \theta^{s}(x)\right)$ could be approximated by following two-scale asymptotic solution in $\Omega_{1}^{\varepsilon}$, 


$$
\left\{\begin{array}{l}
\theta_{\varepsilon}^{(L)}(x)=\theta^{0}(x)+\varepsilon H_{\alpha_{1}}(\xi) \frac{\partial \theta^{0}(x)}{\partial x_{\alpha_{1}}}+\varepsilon^{2} H_{\alpha_{1} \alpha_{2}} \frac{\partial^{2} \theta^{0}(x)}{\partial x_{\alpha_{1}} \partial x_{\alpha_{2}}}+\varepsilon^{3} H_{\alpha_{1} \alpha_{2} \alpha_{3}} \frac{\partial^{3} \theta^{0}(x)}{\partial x_{\alpha_{1}} \partial x_{\alpha_{2}} \partial x_{\alpha_{3}}}+\ldots+\varepsilon^{L} H_{\alpha_{1} \alpha_{2} \ldots \alpha_{L}} D_{\alpha}^{L} \theta^{0}(x), \\
\mathbf{u}_{\varepsilon}^{(L)}(x)=\mathbf{u}^{0}(x)+\varepsilon \mathbf{N}_{\alpha_{1}}(\xi) \frac{\partial \mathbf{u}^{0}(x)}{\partial x_{\alpha_{1}}}+\varepsilon^{2} \mathbf{N}_{\alpha_{1} \alpha_{2}}(\xi) \frac{\partial^{2} \mathbf{u}^{0}(x)}{\partial x_{\alpha_{1}} \partial x_{\alpha_{2}}}+\varepsilon \mathbf{M}_{0}(\xi) \theta^{0}(x)+\varepsilon^{2} \mathbf{M}_{\alpha_{1}}(\xi) \frac{\partial \theta^{0}(x)}{\partial x_{\alpha_{1}}}+\ldots+ \\
\varepsilon^{L} \mathbf{N}_{\alpha_{1} \ldots \alpha_{L}} D_{\alpha}^{L} \mathbf{u}^{0}(x)+\varepsilon^{L-1} \mathbf{M}_{\alpha_{1} \ldots \alpha_{L-1}} D_{\alpha}^{L-1} \theta^{0}(x),
\end{array}\right.
$$

In boundary layer part $\Omega_{2}^{\varepsilon}$, so as to capture the coupling multiple physical effects, we construct the following boundary layer problem,

$$
\left\{\begin{array}{l}
\frac{\partial}{\partial x_{i}}\left(k_{i j}^{\varepsilon} \frac{\partial \theta^{(b, \varepsilon)}}{\partial x_{j}}\right)-c^{\varepsilon} \theta^{(b, \varepsilon)}+h^{\varepsilon}=0, \Omega_{2}^{\varepsilon}, \\
\frac{\partial}{\partial x_{j}}\left(a_{i j h k}^{\varepsilon} \varepsilon_{h k}\left(\mathbf{u}^{(b, \varepsilon)}\right)\right)-\frac{\partial}{\partial x_{j}}\left(a_{i j h k}^{\varepsilon} b_{h k}^{\varepsilon} \theta^{(b, \varepsilon)}\right)-d_{i}^{\varepsilon} u_{i}^{(b, \varepsilon)}+f_{i}^{\varepsilon}(x)=0, \Omega_{2}^{\varepsilon}, \\
\theta^{(b, \varepsilon)}(x)=\theta_{\varepsilon}^{(L)}(x), \mathbf{u}^{(b, \varepsilon)}(x)=\mathbf{u}_{\varepsilon}^{(L)}(x), \text { on } \partial\left(\Omega_{2}^{\varepsilon} \cap \Omega_{1}^{\varepsilon}\right), \\
\theta^{(b, \varepsilon)}(x)=\theta_{0}(x), \mathbf{u}^{(b, \varepsilon)}(x)=\mathbf{u}_{0}(x), \text { on } \Gamma_{\varepsilon} .
\end{array}\right.
$$

Sum up, we have the following formal asymptotic expansion Theorem.

Theorem 1. It is assumed that $a_{i j h k}^{\varepsilon}, b_{h k}^{\varepsilon}, d_{i}^{\varepsilon}, c^{\varepsilon}, k_{i j}^{\varepsilon} \in L^{\infty}(Q), \mathbf{f}^{\varepsilon}(x), \mathbf{u}_{0}(x), h^{\varepsilon}(x), \theta_{0}(x)$ are sufficiently smooth functions in $\Omega^{\varepsilon}$, then:

1) Eq. 1 exists the formal asymptotic expansion of Eq. 2, where $\mathbf{N}_{\alpha}(\xi), \mathbf{M}_{\alpha}(\xi)$ are determined by Eq. 3-Eq. 4, Eq. 5-Eq. 6, the homogenization $\left(\mathbf{u}^{0}(x), \theta^{0}(x)\right)$ could be determined by Eq.9;

2) The homogenization constants $\hat{a}_{i j h}, \hat{b}_{i j}, \hat{d}_{i}, \hat{c}, \hat{k}_{i j}$ could be determined by Eq. 7, Eq. 8 and Eq. 10;

3) Homogenization constants $\hat{b}_{i j}$ satisfy the $p\left(\lambda_{1}, \lambda_{2}\right)$ condition;

4) Homogenization constants $\hat{a}_{i j h}$ satisfy the $\varepsilon\left(\lambda_{1}, \lambda_{2}\right)$ condition;

5) $\left(\mathbf{u}^{\varepsilon}(x), \theta^{\varepsilon}(x)\right)$ could be approximated by following formal two-scale asymptotic solution,

$$
\left(\mathbf{u}^{\varepsilon}(x), \theta^{\varepsilon}(x)\right) \square\left(\mathbf{u}_{\varepsilon}^{(b, L)}(x), \theta_{\varepsilon}^{(b, L)}(x)\right)=\left\{\begin{array}{l}
\left(\mathbf{u}_{\varepsilon}^{(L)}(x), \theta_{\varepsilon}^{(L)}(x)\right), x \in \Omega_{1}^{\varepsilon}, \\
\left(\mathbf{u}^{(b, \varepsilon)}(x), \theta^{(b, \varepsilon)}(x)\right), x \in \Omega_{2}^{\varepsilon} .
\end{array}\right.
$$

\section{The Two-scale Asymptotic Error Estimation}

For the two-scale asymptotic solution, we have the following asymptotic error estimation.

Theorem 2. Assuming that $\theta^{\varepsilon}(x), \mathbf{u}^{\varepsilon}(x)$ are the weak solution of Eq.1, and $\theta_{0}, \mathbf{u}_{0} \in H^{3}(\Omega), \hat{h}$ and $\hat{\mathbf{f}} \in H^{2}(\Omega)$, then the following estimations hold,

$$
\left\|\theta^{\varepsilon}-\theta_{\varepsilon}^{(b, 1)}\right\|_{H^{1}\left(\Omega^{\varepsilon}\right)} \leq c_{1} \varepsilon\left(\left\|\theta_{0}\right\|_{H^{5 / 2}(\Omega)}+\|\hat{h}\|_{H^{2}(\Omega)}\right)
$$

and

$$
\left\|\mathbf{u}^{\varepsilon}-\mathbf{u}_{\varepsilon}^{(b, 1)}\right\|_{H^{1}\left(\Omega^{\varepsilon}\right)} \leq C_{2} \varepsilon\left(\left\|\theta_{0}\right\|_{H^{5 / 2}(\Omega)}+\|\hat{h}\|_{H^{2}(\Omega)}+\left\|\mathbf{u}_{0}\right\|_{H^{5 / 2}(\Omega)}+\|\hat{\mathbf{f}}\|_{H^{2}(\Omega)}\right)
$$


where $c_{1}$ and $c_{2}$ are positive constants independent of $\varepsilon$.

Proof. For simplicity, we only need to prove Eq. 15, Eq.16 could be proved in a similar way. Let $\tilde{\theta}_{\varepsilon}^{(b, 1)}(x)=\theta^{\varepsilon}(x)-\theta_{\varepsilon}^{(b, 1)}(x)$, then after careful computing and deducting, in $\Omega_{1}^{\varepsilon}$ we have the following equations which holds in distribution,

$$
\left\{\begin{array}{l}
\frac{\partial}{\partial x_{i}}\left(k_{i j}^{\varepsilon} \frac{\partial \tilde{\theta}_{\varepsilon}^{(b .1)}}{\partial x_{j}}\right)-c^{\varepsilon} \tilde{\theta}_{\varepsilon}^{(b .1)}=-\left(h^{\varepsilon}-\hat{h}\right)+\varepsilon^{2} F_{1}\left(H_{\alpha}, \theta^{0}\right), x \in \Omega_{1}^{\varepsilon}, \\
\tilde{\theta}_{\varepsilon}^{(b .1)}=0, \text { on } \partial \Omega_{1}^{\varepsilon},
\end{array}\right.
$$

Where $F_{1}\left(H_{\alpha}, \theta^{0}\right)$ is a bounded function independent of $\varepsilon$ and combination of cell functions $H_{\alpha}$ and the 3rd-order partial differentials of $\theta^{0}$.

In $\Omega_{2}^{\varepsilon}$ we have the following equations which holds in distribution,

$$
\left\{\begin{array}{l}
\frac{\partial}{\partial x_{i}}\left(k_{i j}^{\varepsilon} \frac{\partial \tilde{\theta}_{\varepsilon}^{(b .1)}}{\partial x_{j}}\right)-c^{\varepsilon} \tilde{\theta}_{\varepsilon}^{(b .1)}=-\left(h^{\varepsilon}-\hat{h}\right)+\varepsilon^{2} F_{2}\left(H_{\alpha}, \theta^{0}\right), x \in \Omega_{2}^{\varepsilon}, \\
\tilde{\theta}_{\tilde{\theta}}^{(b .1)}=\varepsilon^{2} F_{3}\left(H_{\alpha}, \theta^{0}\right), \text { on } \partial\left(\Omega_{1}^{\varepsilon} \cap \Omega_{2}^{\varepsilon}\right), \\
\tilde{\theta}_{\varepsilon}^{(b .1)}=0, \text { on } \Gamma_{\varepsilon},
\end{array}\right.
$$

Where $F_{2}\left(H_{\alpha}, \theta^{0}\right)$ and $F_{3}\left(H_{\alpha}, \theta^{0}\right)$ are the bounded functions independent of $\varepsilon$ and the combinations of cell functions $H_{\alpha}$ and the 3rd-order partial differentials of $\theta^{0}$. By means of Holder inequalities and generalized Lax-Milgram theorem, the problems Eq. 17 and Eq.18 exist one and only one weak solution in $H^{1}\left(\Omega_{1}^{\varepsilon}\right)$ and $H^{1}\left(\Omega_{2}^{\varepsilon}\right)$, respectively.

By means of the regularities estimations of solutions for Eq.17 and Eq.18, we have:

$$
\left\|\tilde{\theta}_{\varepsilon}^{(b, 1)}\right\|_{H^{1}\left(\Omega_{1}^{\varepsilon}\right)} \leq c_{3}\left(\varepsilon^{2}\left\|F_{1}\left(H_{\alpha}, \theta^{0}\right)\right\|_{H^{1}\left(\Omega_{1}^{\varepsilon}\right)}+\|\hat{h}-h\|_{L^{2}\left(\Omega_{1}^{\varepsilon}\right)}\right)
$$

and

$$
\left\|\tilde{\theta}_{\varepsilon}^{(b, 1)}\right\|_{H^{1}\left(\Omega_{2}^{\varepsilon}\right)} \leq C_{4}\left(\varepsilon^{2}\left\|F_{2}\left(H_{\alpha}, \theta^{0}\right)\right\|_{H^{1}\left(\Omega_{2}^{\varepsilon}\right)}+\varepsilon^{2}\left\|F_{3}\left(H_{\alpha}, \theta^{0}\right)\right\|_{H^{1}\left(\Omega_{2}^{\varepsilon}\right)}+\|\hat{h}-h\|_{L^{2}\left(\Omega_{2}^{\varepsilon}\right)}\right),
$$

where $c_{3}$ and $c_{4}$ are positive constants independent of $\varepsilon$. Owing to properties of periodic functions [5], it is easy to prove that $\|\hat{h}-h\|_{L^{2}\left(\Omega_{1}^{\varepsilon}\right)} \leq c_{5} \varepsilon$, here $c_{5}$ is a positive constant independent of $\varepsilon$. By means of the extension theorem, trace theorem in Sobolev space and regularity estimation of solution for homogenization equations Eq. 9, Eq.15 holds in distribution.

\section{Conclusion}

In this paper, the two-scale asymptotic expansion for one coupling thermoelastic problem in periodic structure with boundary layer is discussed. Because the disturbance of the stiffness, thermal-expansion and thermal conductivity of materials are relatively large and complicated, it is very difficult to obtain the analytic solutions for these equations in mathematics. We try to compute the two-scale asymptotic 
solution and to simulate their numerical solution. In order to improve the accuracy of the calculation, we construct the matched boundary layer problem. In addition, some asymptotic error estimates are given, which show the effective of the expansion. In this paper, we use two-scale method to construct the formal asymptotic expansion for thermoelastic problem with general items and with boundary layer, what is more, we improve the asymptotic error estimation order from $\varepsilon^{1 / 2}$ to $\varepsilon$.

The results could be regarded as the reference for evaluating the effective physical mechanic and thermal behavior. More, our results could be useful for designing the numerical algorithm and computing the approximate solutions for these coupling systems in multiple fields.

\section{Acknowledgment}

The research is supported by the Science and Technology Project for the Colleges and Universities of Guangzhou Education Bureau [Grant: 1201430652].

\section{References}

[1] Feng Y.P., Deng M.X., Guan X.F., Cui J.Z., A Two-scale finite element analysis of the thermo-elastic effects in composites, International Journal of Computational Method. 11(2014) 1350066.

[2] Chen J.R., Cui J.Z., Two-scale finite element method for non-self-adjoint elliptic problems with rapidly oscillatory coefficients, Appl. Math. Comp. 150(2004)585-601.

[3] Du R., Ming P.B., Convergence of the heterogeneous multi-scale finite element method for elliptic problem with non-smooth microstructure, Multiscale Model. Simul. 8(2010)1770 -1783.

[4] Han F., Cui J.Z., Yu Y., The statical second-order two-scale method for thermo-mechanical properties of statistically inhomogeneous materials, Computational Materials Sciences. 46(2009)654-659.

[5] Cao L.Q., Cui J.Z., Homogenization method for the quasi-periodic structures of composite materials, Math. Numer. Sinica. 21(1999)333-342. 